Check for updates

Cite this: Mater. Adv., 2022, 3,570

Received 2nd September 2021, Accepted 3rd November 2021

DOI: 10.1039/d1ma00801c

rsc.li/materials-advances

\section{White light emitting silsesquioxane based materials: the importance of a ligand with rigid and directional arms $\dagger$}

\author{
Andrea Santiago-Portillo, ${ }^{a}$ Valerio Cinà, ${ }^{\text {ab }}$ Esther Carbonell, ${ }^{a}$ Luca Fusaro, (D) ${ }^{a}$ \\ Vincent Lemaur, (iD c Roberto Lazzaroni, (iD c Michelangelo Gruttadauria, (iD b \\ Francesco Giacalone (D) *b and Carmela Aprile (D) *a
}

\begin{abstract}
The synthesis of a novel polyhedral oligomeric silsesquioxane functionalized with eight rigid and directional terpyridine-based arms (Ter-POSS) was successfully achieved via a Sonogashira reaction. The POSS based ligand was extensively characterized using different techniques including ${ }^{1} \mathrm{H},{ }^{13} \mathrm{C}$ and ${ }^{29} \mathrm{Si}$ NMR as well as UV-Vis and fluorescence spectroscopies. The assembly of these nano-caged units in the presence of different transition metal ions $\left(\mathrm{Fe}^{2+}, \mathrm{Zn}^{2+}\right.$ and $\left.\mathrm{Cu}^{2+}\right)$ as well as of a cation from the lanthanides $\left(\mathrm{Eu}^{3+}\right)$ was investigated using absorption and emission spectroscopies. The final materials display an evident emission in different regions of the visible spectrum as a function of the cation employed. Additional insights into the structural organization of Ter-POSS in the presence of metal cations were obtained via molecular mechanics and molecular dynamics simulations. The polymeric material resulting from the complexation with europium displays a white light emission ascribed to the presence of combined contributions from the blue, green and red regions. The final self-assembled organizations display an increased quantum yield with the highest value $(29.6 \%)$ obtained in the presence of $\mathrm{Zn}^{2+}$. Moreover, the white-light emitting europium-based nanostructure exhibits one of the highest quantum yields reported in the literature for similar solids.
\end{abstract}

\section{Introduction}

Nowadays, structured organic-inorganic hybrid solids play an important role in the development of functional (nano)materials. Nano-caged molecules such as polyhedral oligomeric silsesquioxanes (POSS) represent an exceptional building unit since they merge the characteristics of the organic moieties and the inorganic core into one nano-entity. ${ }^{1}$ The applications involving the use of POSS cover a wide range of scientific fields including optics, photonics, electronics, catalysis, sensors and biology. $^{2-7}$ This broad window of possible applications is mainly related to the tuneable features of the nanocages as a function of the nature of the organic corners together with the

\footnotetext{
${ }^{a}$ Unit of Nanomaterial Chemistry (CNano), Department of Chemistry, University of Namur, Rue de Bruxelles 61, 5000, Namur, Belgium. E-mail: carmela.aprile@unamur.be

${ }^{b}$ Department of Biological, Chemical and Pharmaceutical Sciences and Technologies, University of Palermo, Viale delle Scienze, Ed.17, 90128, Palermo, Italy. E-mail: francesco.giacalone@unipa.it

${ }^{c}$ Laboratory for Chemistry of Novel Materials, Materials Research Institute, University of Mons-UMONS, Place du Parc 20, Mons B-7000, Belgium

$\dagger$ Electronic supplementary information (ESI) available. See DOI: 10.1039/d1ma00801c
}

enhanced mechanical and thermal stabilities often provided by the inorganic core.

Recently, novel ionic liquids constituted by properly functionalized anionic POSS and imidazolium-based moieties as cation species were synthesized. It was found that the introduction of a POSS moiety improves the thermal stability while decreasing the melting temperature. ${ }^{8}$ POSS structures have been also employed to create novel polymeric materials. ${ }^{9}$ Wang et al. reported a method to produce inorganic-organic hybrid hydrogels via controlled cross-linking reactions. ${ }^{10}$ Ghanbari et al. described the synthesis of nanocomposite materials obtained by copolymerization of POSS monomers with poly(carbonate urea)urethane (POSS-PCU) to form covalently modified and cross-linked nanostructures used for biomedical devices. ${ }^{11}$ Qiu et al. reported the use of trisilanolisobutyl-POSS (tsib-POSS) to strengthen the mechanical properties of a poly(L-lactide) (PLLA) nanocomposite. ${ }^{12}$

Interestingly, POSS-based structures were also employed for photophysical applications. An octaimidazolium-based POSS bearing a lanthanide complex as counterion was efficiently prepared by ion exchange. ${ }^{13}$ The authors claimed that the presence of the silsesquioxane nanocage allows enhancing both the fluorescence intensity and the lifetime. The functionalization 
of silsesquioxanes with terpyridines ${ }^{14-19}$ was also explored for the synthesis of materials with tuneable photophysical properties. We recently reported the synthesis of terpyridine functionalized POSS in which the organic units were connected to the silsesquioxane via an alkenyl group. Tuneable emissions were achieved via trans-cis isomerization of the double bond. ${ }^{20,21}$

It has been demonstrated that the presence of POSS can significantly increase both the lifetime and thermal stability of the compounds used as emitters in light emitting diode (LED). ${ }^{22-26}$ At this juncture, it is worth to mention that the field of LED and in particular white light emitting diodes (WLEDs) have attracted particular attention. White light materials are often prepared from combinations of several components displaying complementary emission colours such as blue and yellow (BY), blue and red (BR) or, more recently, blue, green and red (BGR) ${ }^{27}$ Organic materials are most widely used in the synthesis of WLED. ${ }^{28-30}$ Most organic WLEDs are synthetized by mixing different organic polymers with unique optical properties. However, they can often suffer from deactivation, poor colour reproduction and short lifetimes. ${ }^{28,31}$ Interestingly, there are few examples where properly functionalized POSS have been used as white light emitting materials. Zhao et al. reported the preparation of single molecular POSS-based whitelight (WL) emitting hybrid materials synthesized through a click chemistry approach. WL emission was achieved by controlling the feed ratio of blue and yellow emitting units. ${ }^{32}$ In another study, Yang et al. obtained WL emitting materials employing POSS units functionalized with iridium complexes. In this work an increased stability of the WL emitting solid with a quantum yield (QY) of $8 \%$ was reported. ${ }^{33-35}$

Herein, the synthesis of a novel POSS-based ligand bearing rigid arms terminated with terpyridine moieties is reported (TerPOSS). Ter-POSS behaves as an efficient ligand for various transition metal cations such as iron, zinc and copper forming coordination polymers displaying violet, cyan or green emission..$^{20,21,36}$ Moreover, in the presence of a cation from the family of lanthanides $\left(\mathrm{Eu}^{3+}\right)$, a white emission with a corresponding QY of $12 \%$ was achieved. The WL emission was ascribed to the presence of rigid and directional arms limiting the intra-molecular interactions between the terpyridine units and thus favouring the presence of a combination of BGR emitting colours. It should be highlighted that this combination of BGR emissions did not require a complex mixture of different emitting partners and was obtained using a single component ligand. Interestingly, the final materials arrange in polymeric architectures as indicated via transmission electron microscopy investigation. The simplicity of the synthesis procedure together with the versatility of the novel POSS-based ligand to be able to produce polymers emitting in different regions of the visible spectrum and with elevated QY makes this approach highly promising in view of the applications in the field of LED including WLED.

\section{Results and discussion}

A novel POSS-based nanostructure bearing directional and rigid arms terminating with terpyridine moieties was successfully a)

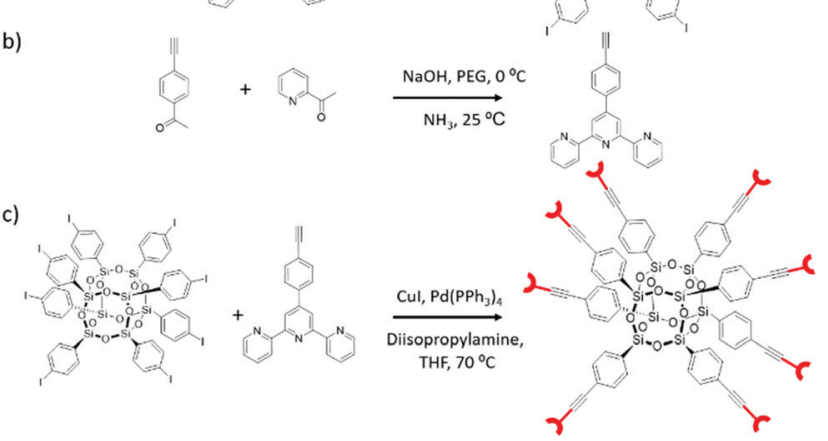

Scheme 1 (a) Synthesis of I-POSS, (b) synthesis of 4' -(4-ethylnylphenyl)$\left[2,2^{\prime}: 6,2^{\prime \prime}\right]$ terpyridine, and (c) synthesis of novel POSS (Ter-POSS) via a Sonogashira reaction.

obtained following the synthesis strategy depicted in Scheme 1. As indicated in the scheme, the commercially available octaphenyl-POSS (Ph-POSS in Scheme 1a) was selected as the starting material. The first step involved the iodination of Ph-POSS with ICl at $-40{ }^{\circ} \mathrm{C}^{37}$ Under the selected reaction conditions, the iodination reaction was highly regioselective towards substitution in the para-position of the aromatic ring (I-POSS). The full characterization of I-POSS via ${ }^{1} \mathrm{H},{ }^{13} \mathrm{C}$ and ${ }^{29} \mathrm{Si}$ NMR allows confirming the purity of the compound. In agreement with the data reported in the literature, ${ }^{37}$ the ${ }^{1} \mathrm{H}$ NMR spectrum (Fig. 1a) displays two singlets in the aromatic region, while the ${ }^{13} \mathrm{C}$ NMR spectrum (Fig. S1, ESI $\dagger$ ) presents two main signals at $\delta=135$ and $137 \mathrm{ppm}$. As expected, the ${ }^{29} \mathrm{Si} \mathrm{NMR}$ of I-POSS (Fig. S2, ESI $\dagger$ ) shows the presence of one narrow contribution at $c a .-78 \mathrm{ppm}$ which can be assigned to the completely condensed $\mathrm{RSi}(\mathrm{OSi})_{3}\left(\mathrm{~T}_{3}\right)$ species indicative of a closed $\mathrm{T}_{8} \mathrm{R}_{8}$ structure.

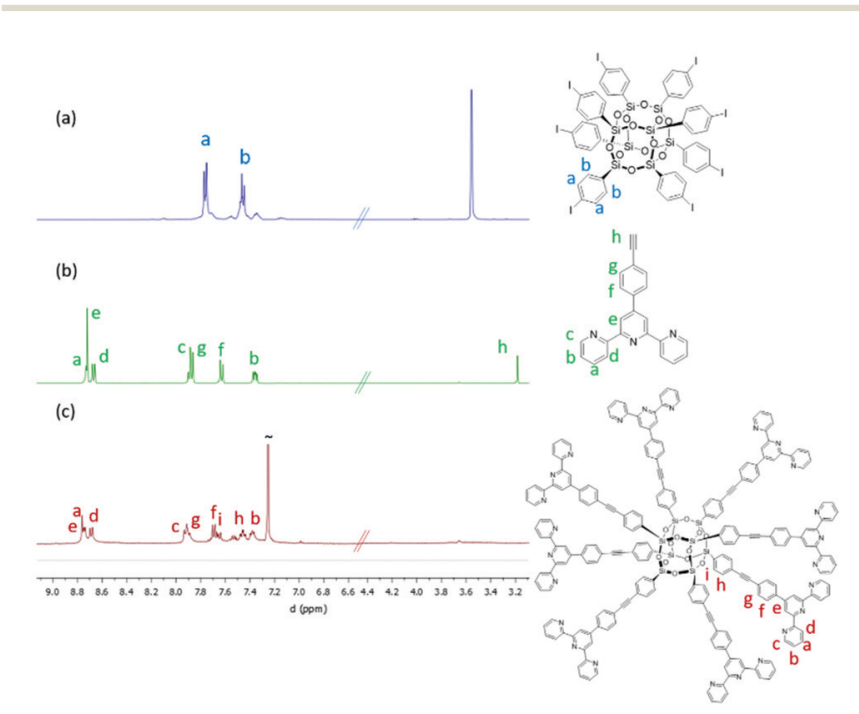

Fig. $1{ }^{1} \mathrm{H}$ NMR spectra of (a) I-POSS, (b) 4'-(4-ethylnylphenyl)$\left[2,2^{\prime}: 6,2^{\prime \prime}\right]$ terpyridine and (c) Ter-POSS. 
In order to proceed with the following step, based on a Sonogashira coupling reaction, the synthesis of $4^{\prime}$-(4-ethylnylphenyl)-[2,2' $\left.: 6,2^{\prime \prime}\right]$ terpyridine (Terpy) was performed following a procedure described in the literature (Scheme 1b). ${ }^{19}$ The structure and purity of the final compound were confirmed via ${ }^{1} \mathrm{H}$ and ${ }^{13} \mathrm{C}$ NMR experiments (Fig. S3 and S4, ESI $\dagger$ ) as well as via combustion chemical analysis.

Finally, the synthesis of the novel POSS functionalized with terpyridine moieties (Ter-POSS) was achieved (yield 80\%) via a Sonagashira cross-coupling reaction between I-POSS and Terpy in the presence of tetrakis(triphenylphosphine) palladium (as the catalyst) and copper iodide (as the co-catalyst) (Scheme 1c).

The final solid was extensively characterized using ${ }^{1} \mathrm{H},{ }^{13} \mathrm{C}$, ${ }^{29} \mathrm{Si}$ NMR, UV-Vis and IR spectroscopies as well as via combustion chemical analysis. The complete assignment of the ${ }^{1} \mathrm{H}$ NMR signals was achieved via the homonuclear 2D NMR experiment (Fig. S5, ESI $\dagger$ ). The quantitative ${ }^{1} \mathrm{H}$ NMR spectra of Ter-POSS together with those of I-POSS and Terpy are presented in Fig. 1. The good performance of the reaction is confirmed by the disappearance of the singlet at $3.2 \mathrm{ppm}$ accompanied by the slight shift of the signals in the aromatic region. It should be highlighted that the pattern of signals in the ${ }^{1} \mathrm{H}$ NMR spectra is not affected by the strong broadening previously observed for other octa-functionalized silsesquioxanes ${ }^{20}$ thus indicating the absence of intra-molecular $\pi-\pi$ stacking interactions between the aromatic moieties. ${ }^{17,24}$ The correct assignment of the chemical shifts was achieved via ${ }^{1} \mathrm{H}-{ }^{1} \mathrm{H}$ homonuclear NMR correlation spectroscopy (COSY) presented in Fig. S5 (ESI $\dagger$ ).

Due to the low solubility of Ter-POSS, both ${ }^{13} \mathrm{C}$ and ${ }^{29} \mathrm{Si}$ NMR spectroscopies were performed in the solid state. The ${ }^{13} \mathrm{C}$ CP-MAS NMR spectra of Ter-POSS was characterized by a pattern of signals in the aromatic region corresponding to the overlapping of the different contributions as indicated in Fig. S6 (ESI $\dagger$ ). More interestingly, the ${ }^{29} \mathrm{Si}$ CP-MAS NMR spectrum of Ter-POSS shows the presence of one main signal centred at $c a . \delta=-86 \mathrm{ppm}$, indicating the presence of a closed $\mathrm{T}_{8} \mathrm{R}_{8}$ structure corresponding to the completely condensed $\mathrm{T}^{3}$ units thus confirming the stability of the silsesquioxane core (Fig. S7, ESI $\dagger$ ).

To gain further insights on the purity of the compound, combustion chemical analysis of Ter-POSS was carried out. The results obtained (see the Experimental section) confirm the good correlation between the experimental and theoretical values.

The IR spectrum of Ter-POSS (Fig. S8, ESI $\dagger$ ) displays the typical fingerprint of the closed $\mathrm{T}_{8} \mathrm{R}_{8}$ POSS nanocage with an intense band centred at $c a .1000 \mathrm{~cm}^{-1}$ which is attributed to the Si-O-Si symmetric stretching together with two minor contributions at around $800 \mathrm{~cm}^{-1}$ and $470 \mathrm{~cm}^{-1}$ which can be assigned to the $\mathrm{Si}-\mathrm{O}-\mathrm{Si}$ asymmetric stretching and rocking modes, respectively.

The absorption and emission properties of Ter-POSS were characterized as well, via UV-Vis and fluorescence spectroscopies (Fig. 2). The UV-Vis spectrum shows two main absorption bands with maxima at 290 and $310 \mathrm{~nm}$, respectively, which can be attributed to the terpyridine ligands. ${ }^{20}$ The fluorescence spectrum of Ter-POSS displays a broad emission band that can
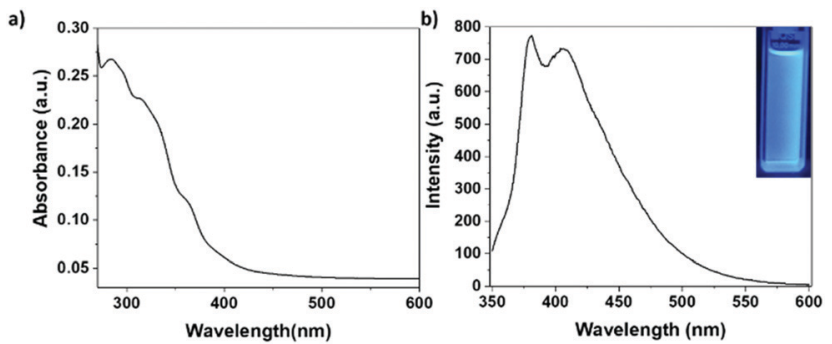

Fig. 2 Absorption and emission spectra of Ter-POSS in $\mathrm{CH}_{2} \mathrm{Cl}_{2}\left(1 \times 10^{-6}\right.$ M); $\lambda_{\mathrm{ex}}=330 \mathrm{~nm}, \mathrm{OD}=0.23$, and slits $=5 \mathrm{~nm}$.

be considered as the result of two partially overlapping contributions centred at 380 and $420 \mathrm{~nm}$. As can be seen in the inset of Fig. 2b, under UV light exposure Ter-POSS displays an intense blue emission. In agreement with the literature, ${ }^{20}$ these bands were assigned to the typical emission of the terpyridine moieties. The slight broadening of these bands toward longer wavelength could be reasonably ascribed to the possible presence of inter-molecular excimers, which are present only to a minor extent in solution due to the high dilution of the medium. The reduced presence of excimers is also supported by the intensity of the emission band, confirming the absence of the self-quenching phenomena. ${ }^{17}$

To further support the previous findings and gain additional insights into the structural organization of Ter-POSS in the presence of metal cations, molecular mechanics and molecular dynamics simulations were performed with a force field derived from the Dreiding force field (see details in the Experimental section). The most stable structure of Ter-POSS exhibits a fully extended conformation with its eight arms pointing in the opposite directions (Fig. 3). The closest distance between the central pyridine ring of different arms is never below $17 \AA$, as demonstrated by a radial distribution function analysis on a 10 ns molecular dynamics simulation (see Fig. S9, ESI $\dagger$ ). This confirms that there are no intramolecular $\pi-\pi$ interactions in Ter-POSS and, consequently, supports the hypothesis of the absence of intra-molecular excimers proposed from the ${ }^{1} \mathrm{H}$ NMR and fluorescence spectroscopy data.

This situation contrasts with the octa-functionalized POSS structures previously synthesized by our group in which more flexible chains were connecting the aromatic moieties to the silsesquioxane core (O-POSS). ${ }^{20,21}$ As it can be seen in Fig. S10 (ESI $\dagger$ ), both $Z$ and $E$ isomers of O-POSS display significant intramolecular $\pi-\pi$ interactions in their more stable forms, which gives rise to partial fluorescence quenching. This comparison points out the crucial role played by the chemical nature of the linker between the terpyridine groups and the POSS cage, which can be probed by numerical simulations prior to any synthesis. This combination of experimental and theoretical studies therefore provides a strong background for molecular design to form tailored supramolecular structures via metalligand (ML) interactions.

These ML interactions could be studied by ${ }^{1} \mathrm{H}$ NMR spectroscopy. However, as mentioned previously, due to the low solubility of Ter-POSS in various solvents this technique cannot 


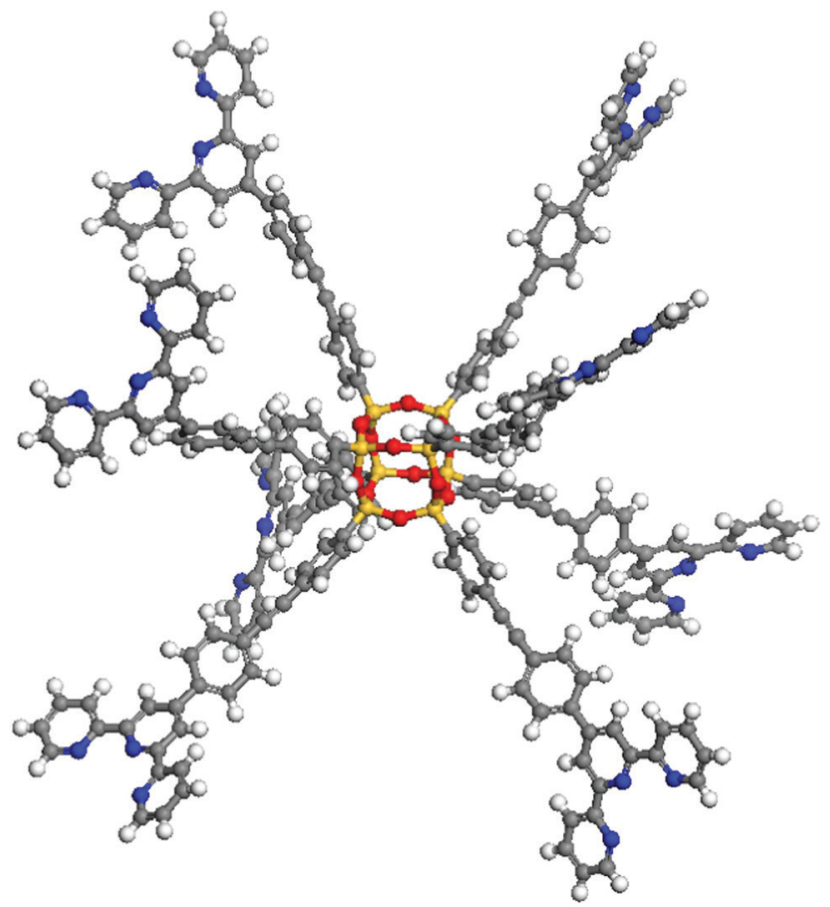

Fig. 3 Representation of the most stable structure of Ter-POSS with its directional ligands (the terpyridine moieties are forced to assume an arrangement simulating the coordination with a metal cation).

be applied herein. For this reason, quantitative titration experiments were performed via both UV-Vis and emission spectroscopies. These experiments allow obtaining information on the stoichiometry of the complexes formed and on the photophysical properties of the final structures.

It is known from the literature that terpyridine moieties display excellent complexation properties for a wide range of metal ions. ${ }^{20,21}$ Three different transition metal cations $\left(\mathrm{Fe}^{2+}\right.$, $\mathrm{Zn}^{2+}$ and $\mathrm{Cu}^{2+}$ ) and one cation from the family of lanthanides $\left(\mathrm{Eu}^{3+}\right)$ were selected in this study.

Fig. 4 presents the results of the titration of Ter-POSS upon adding increasing amount of iron(II) trifluoromethanesulfonate $\left(\mathrm{Fe}(\mathrm{OTf})_{2}\right)$. As expected, in the UV-visible spectra a new band due to the $\mathrm{Fe}^{2+}$ to ligand interaction appears. This band centred
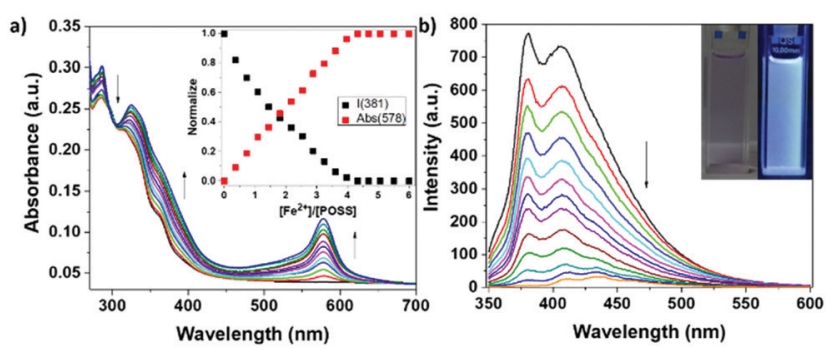

Fig. 4 (a) UV-Vis absorption spectra of Ter-POSS in $\mathrm{CH}_{2} \mathrm{Cl}_{2}\left(1 \times 10^{-6} \mathrm{M}\right)$ upon titration with $\mathrm{Fe}(\mathrm{OTF})_{2}$ in $\mathrm{EtOH}\left(3.63 \times 10^{-4} \mathrm{M}\right)$. The inset shows the normalized absorption changes at $574 \mathrm{~nm}$ (red squares) and the normalized emission intensity changes at $380 \mathrm{~nm}$ (black squares). (b) Emission spectra of Ter-POSS in $\mathrm{CH}_{2} \mathrm{Cl}_{2}\left(1 \times 10^{-6} \mathrm{M}\right)$ upon titration with $\mathrm{Fe}(\mathrm{OTF})_{2}$ in $\mathrm{EtOH}\left(3.63 \times 10^{-4} \mathrm{M}\right) ; \lambda_{\mathrm{ex}}=330 \mathrm{~nm}, \mathrm{OD}=0.23$, and slits $=5 \mathrm{~nm}$. at $578 \mathrm{~nm}$ is typical of MLCT (metal to ligand charge transfer). In addition, a red-shift of the terpyridine absorption band at $310 \mathrm{~nm}$ with an isosbestic point at $330 \mathrm{~nm}$ was evidenced. Upon excitation at the isosbestic point, the emission band was almost completely quenched. It should be noted that, despite the decrease in the intensity of the emission, the Ter-POSS@4Fe solution is characterized by a violet colour typical of the $\mathrm{Fe}(\mathrm{II})$ complexes (inset of Fig. $4 \mathrm{~b}$ ).

The plots of the normalized variation of the intensity of the absorption band centred at $c a .578 \mathrm{~nm}$ and the intensity of the emission band centred at $380 \mathrm{~nm}$ as a function of the metal to ligand ratio (see inset of Fig. 4a), display a plateau after the addition of 4 equiv. of $\mathrm{Fe}(\mathrm{OTf})_{2}$. These results prove that 4 equiv. of metal cation are required to completely coordinate the eight terpyridine moieties of the POSS-based ligand and indicate that a 4:1 metal to ligand stoichiometry is reached at the end of the titration, with the consequent formation of the Ter-POSS@4Fe complex.

It deserves to be mentioned that no further changes in the UV-Vis spectra were observed after the addition of an excess of metal cation (till 6 equivalents) indicating that the stoichiometry of the complex formed is preserved even at high metal loading in solution.

This result confirms the stability of the Ter-POSS@4Fe architecture and the elevate complexing properties of the ligand selected.

Analogous experiments were performed employing zinc trifluoromethanesulfonate $\left(\mathrm{Zn}(\mathrm{OTf})_{2}\right)$ as the source of the metal cation (Fig. 5). Also, in this case, important changes in the UV-visible absorption bands can be easily detected. As described previously in the presence of $\mathrm{Fe}^{2+}$, a red shift of the terpyridine absorption band centred at $310 \mathrm{~nm}$ and an isosbestic point at $330 \mathrm{~nm}$ were observed. Upon excitation at the isosbestic point, a complete quenching of the emission bands at 380 and $420 \mathrm{~nm}$ was discerned. However, in this case a new band centred at ca. $500 \mathrm{~nm}$ appeared due to the formation of the complex. The presence of this band was accompanied by a clear shift in the emission colour from blue to cyan. Similar to the previous experiments, the plots of the normalized variation of the intensity of the emission and absorption bands attained a plateau in

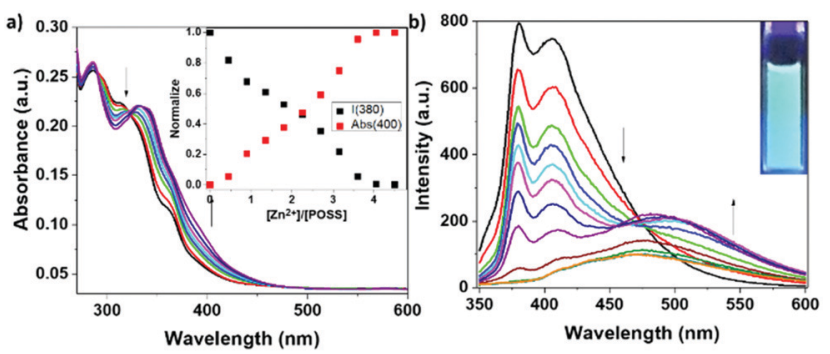

Fig. 5 (a) UV-Vis absorption spectra of Ter-POSS in $\mathrm{CH}_{2} \mathrm{Cl}_{2}\left(1 \times 10^{-6} \mathrm{M}\right)$ upon titration with $\mathrm{Zn}(\mathrm{OTF})_{2}$ in $\mathrm{EtOH}\left(4.18 \times 10^{-4} \mathrm{M}\right)$. The inset shows the normalized absorption changes at $400 \mathrm{~nm}$ (red squares) and the normalized emission intensity changes at $380 \mathrm{~nm}$ (black squares). (b) Emission spectra of Ter-POSS in $\mathrm{CH}_{2} \mathrm{Cl}_{2}\left(1 \times 10^{-6} \mathrm{M}\right)$ upon titration with $\mathrm{Zn}(\mathrm{OTF})_{2}$ in $\mathrm{EtOH}\left(4.18 \times 10^{-4} \mathrm{M}\right) ; \lambda_{\mathrm{ex}}=330 \mathrm{~nm}, \mathrm{OD}=0.22$, and slits $=5 \mathrm{~nm}$. 

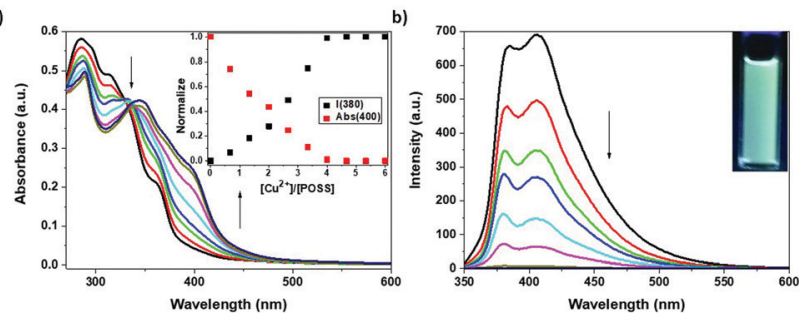

Fig. 6 (a) UV-Vis absorption spectra of Ter-POSS in $\mathrm{CH}_{2} \mathrm{Cl}_{2}\left(1 \times 10^{-6} \mathrm{M}\right)$ upon titration with $\mathrm{Cu}(\mathrm{OTF})_{2}$ in $\mathrm{EtOH}\left(4.18 \times 10^{-4} \mathrm{M}\right)$. The inset shows the normalized absorption changes at $400 \mathrm{~nm}$ (red squares) and the normalized emission intensity changes at $380 \mathrm{~nm}$ (black squares). (b) Emission spectra of Ter-POSS in $\mathrm{CH}_{2} \mathrm{Cl}_{2}\left(1 \times 10^{-6} \mathrm{M}\right)$ upon titration with $\mathrm{Cu}(\mathrm{OTF})_{2}$ in $\mathrm{EtOH}\left(4.18 \times 10^{-4} \mathrm{M}\right) ; \lambda_{\mathrm{ex}}=330 \mathrm{~nm}, \mathrm{OD}=0.38$, and slits $=5 \mathrm{~nm}$.

correspondence of 4 equivalents of $\mathrm{Zn}^{2+}$ hence indicating that a Ter-POSS@4Zn species was formed.

Due to the importance of the development of novel emitting solids with easily tuneable fluorescence, we decided to extend the investigation to another largely studied metal cation: $\mathrm{Cu}^{2+}$. For the sake of coherence with the previous experiments, copper(II) trifluoromethanesulfonate $\left(\mathrm{Cu}(\mathrm{OTf})_{2}\right)$ was selected as the titrating agent (Fig. 6). In this case, upon addition of increasing amounts of $\mathrm{Cu}^{2+}$ to a solution of the silsesquioxanebased ligand, a new MLCT band centred at $c a .400 \mathrm{~nm}$ appeared in the UV-Vis spectra. This band was attributed to the formation of a Cu-terpyridine complex (Ter-POSS@4Cu). After excitation at the isosbestic point $(330 \mathrm{~nm})$, an almost complete quenching of the emission bands at 380 and $420 \mathrm{~nm}$ was observed.

Surprisingly, despite the almost complete disappearance of the emission bands, at the end of the titration experiment the solution displayed a nice light-green colour. A careful look at the emission spectra allows evidencing the appearance of a novel and weak emission band centered at $c a .520 \mathrm{~nm}$ (Fig. S18, ESI $\dagger$ ) responsible for the emission in green. The formation of the Ter-POSS@4Cu complex can be assessed also in this case from the plot of the normalized intensities of the absorption and emission bands as a function of the $M / L$ ratio (inset of Fig. 6).

Fig. 7 represents a schematic illustration of Ter-POSS@4Fe, Ter-POSS@4Zn or Ter-POSS@4Cu. The structure depicted in Fig. 7 allows to envisage the possible development of a 3D ordered architecture; however, due to steric constraints a 3D (or even a lower dimensional) branched polymeric network can appear more probable. As previously mentioned in the introduction, the synthesis of functional emitting polymers is highly demanding.

For this reason and to further extend the scope of this work, the investigation of the POSS-based ligand was extended to one cation from the family of lanthanides. $\mathrm{Eu}^{3+}$ was selected for this purpose due to the well-known intense line-like emission from the f-f electronic transitions at 580, 591, 617, 650 and $698 \mathrm{~nm}$. No relevant differences with respect to the previously described behaviour were observed in the UV-Vis spectra upon the addition of increasing amounts of $\mathrm{Eu}^{3+}$ to the Ter-POSS solution. Upon excitation at the isosbestic point, a complete quenching of the emission band at $380 \mathrm{~nm}$ was observed. As expected,

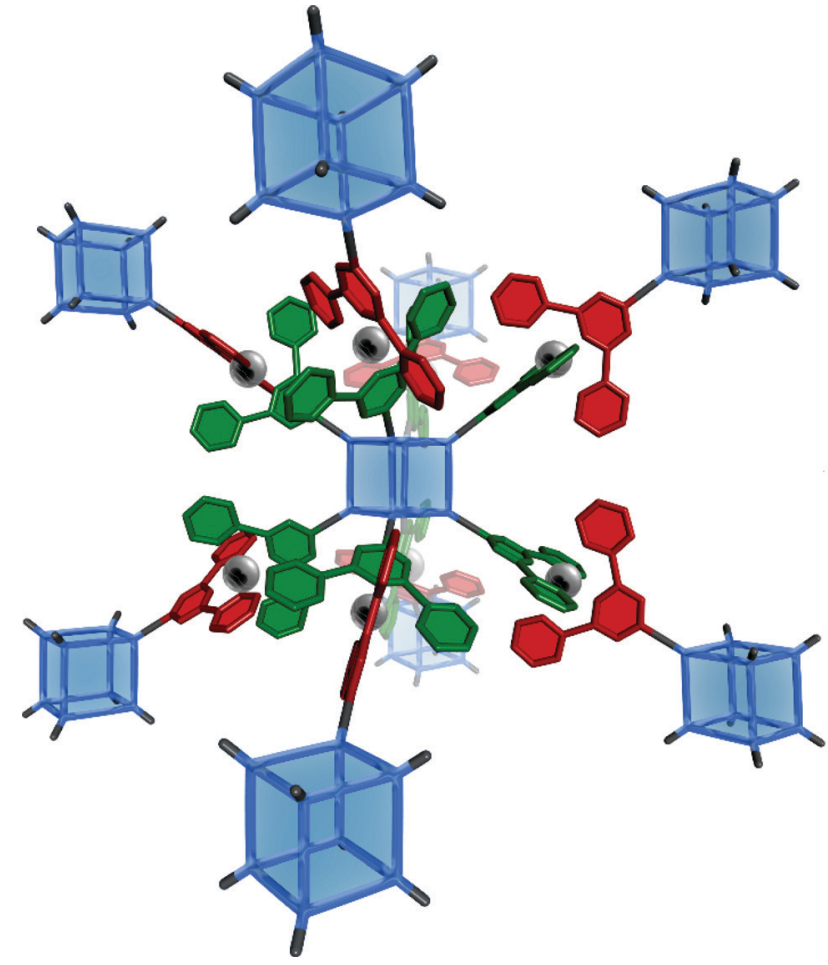

Fig. 7 Schematic representation of the complexes Ter-POSS@4Fe, Ter-Poss@4Zn or Ter-POss@4Cu.

a series of bands typical of the line-like emission of the complexed europium cations centred at 580, 591, 617 and $650 \mathrm{~nm}$ can be clearly distinguished. The normalized plots of intensity of the absorption band centred at $400 \mathrm{~nm}$ and the emission band centred at $380 \mathrm{~nm}$, show a plateau in correspondence to 4 equivalents of the cation. This result indicates that the Ter-POSS@4Eu complex is formed and suggests that the coordination shell of the lanthanide cation is most probably completed by solvent molecules or by the counterion of the salts used for the titration. This behaviour is not surprising and can be ascribed to the large steric hindrance of the POSS units, hampering the simultaneous presence of the three POSS-based ligands in the coordination shell of $\mathrm{Eu}^{3+} \cdot{ }^{18}$ Interestingly, Ter-POSS@4Eu displays a white light emission. This behaviour was previously observed in the presence of ultra-small europium nanocrystals and was ascribed to the combination of blue, green and red emissions. ${ }^{27}$ In our case, the absence of the broad band due to formation of excimers allows evidencing the two contributions at $c a .400$ and $500 \mathrm{~nm}$. These two contributions (in the blue and green regions of the visible spectrum) together with those of the europium complex (with its typical emission in the red region) can explain the resulting intense white light emission.

It is important to mention that ethanol does not display any competitive interaction with the terpyridine moieties. As can be seen in Fig. 4, 5, 6 and 8, when metal cations are added to the solution containing Terpy-POSS a new band immediately appeared in the adsorption and emission spectra. This band was attributed to the specific interaction between the metal 

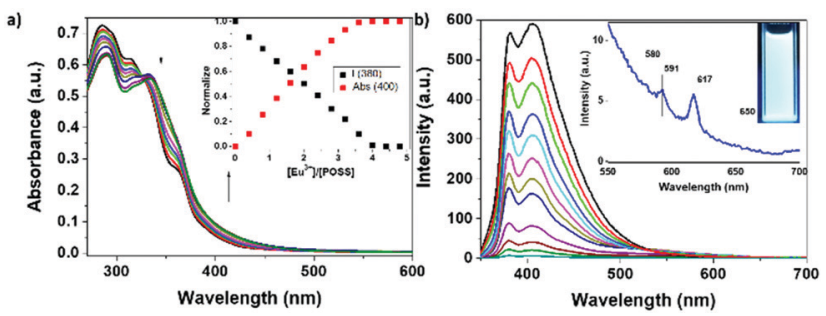

Fig. 8 (a) UV-Vis absorption spectra of Ter-POSS in $\mathrm{CH}_{2} \mathrm{Cl}_{2}\left(1 \times 10^{-6} \mathrm{M}\right)$ upon titration with europium(III) trifluoromethane-sulfonate $\left(\mathrm{Eu}(\mathrm{OTF})_{3}\right)$ in $\mathrm{EtOH}\left(4.18 \times 10^{-4} \mathrm{M}\right)$. The inset shows the normalized absorption changes at $400 \mathrm{~nm}$ (red squares) and the normalized emission intensity changes at $380 \mathrm{~nm}$ (black squares). (b) Emission spectra of Ter-POSS in $\mathrm{CH}_{2} \mathrm{Cl}_{2}$ $\left(1 \times 10^{-6} \mathrm{M}\right)$ upon titration with $\mathrm{Eu}(\mathrm{OTF})_{3}$ in $\mathrm{EtOH}\left(4.18 \times 10^{-4} \mathrm{M}\right) ; \lambda_{\mathrm{ex}}=$ $330 \mathrm{~nm}, \mathrm{OD}=0.55$, and slits $=5 \mathrm{~nm}$

cation employed and the terpyridine-based ligands. Moreover, in previous studies concerning terpyridine-based moieties, the titration experiments were followed also using ${ }^{1} \mathrm{H}$ NMR. In any case a preferential (respect to metal cation) interaction between the terpyridine moieties and alcohols used as the solvent was observed. $^{20,21}$

This result further supports the importance of the design of a POSS-based ligand displaying rigid and directional terpyridine-based arms. Moreover, the simplicity of the synthesis procedure together with the versatility of the nanocage as the ligand makes this structure particularly interesting as a building unit for the synthesis of emitting polymers.

To shed more light on the properties of the emitting materials, the quantum yield was calculated for all the samples using quinine sulphate and rhodamine 101 as standards (see Fig. S11-S22, ESI $\dagger$ ). ${ }^{38}$ As evidenced from the analysis of the data reported in Table 1, all the complexes (except Ter-POSS@4Cu) display high quantum yields with the highest value presented by Ter-POSS@4Zn. Interestingly, the sample with $\mathrm{Eu}^{3+}$ also presents a large quantum yield $(11.8 \%)$ compared to those reported in the literature. ${ }^{39}$ Shunmugan et al. performed the synthesis of Ln-terpyridine complexes and with $\mathrm{Eu}$ they obtained a quantum yield of $1 \%$; this quantum yield was higher $(4.6 \%)$ when they obtained a white light emitting material by mixing different lanthanides. ${ }^{39}$ This enhanced QY can be ascribed to the excellent complexing properties of the terpyridine and to the absence of $\pi-\pi$ stacking interactions with the consequent self-quenching phenomena. ${ }^{27}$

The low QY observed for Ter-POSS@4Cu is in agreement with the one reported in the literature and can be justified by

Table 1 Quantum yield obtained for Ter-POSS, Ter-POSS@4Fe, Ter-POSS@4Zn, Ter-POSS@4Cu and Ter-POSS@4Eu

\begin{tabular}{ll}
\hline POSS & Quantum yield $(\%)$ \\
\hline Ter-POSS & 12.2 \\
Ter-POSS@4Fe & 12.2 \\
Ter-POSS@4Zn & 29.6 \\
Ter-POSS@4Cu & 2.2 \\
Ter-POSS@4Eu & 11.8
\end{tabular}
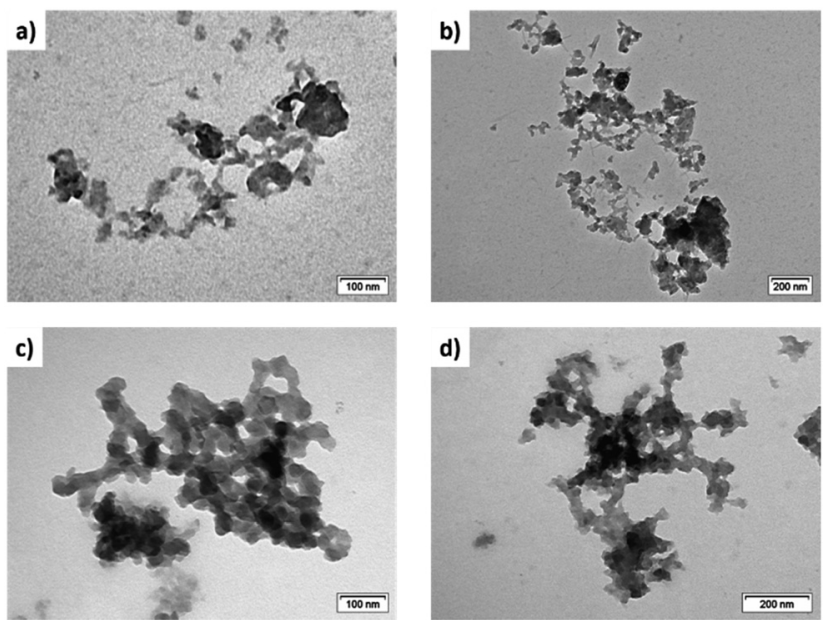

Fig. 9 Transmission electron microscopy images of lyophilized Ter-POSS ( $\mathrm{a}$ and $\mathrm{b}$ ) and Ter-POSS (a 4Fe (c and d).

the almost complete quenching of the emission band of the ligand. ${ }^{40}$ The slight emission is attributed to the appearance of the novel and less intense band centered at $c a .520 \mathrm{~nm}$, which is responsible for the green emission (Fig. S17, ESI $\dagger$ ).

It deserves to be mentioned that the terpyridine-functionalized silsesquioxanes present good thermal stability and photostability as previously demonstrated via $E-Z$ isomerization cycles induced via thermal or photochemical treatments ${ }^{21}$ To demonstrate the formation of polymeric organizations, transmission electron microscopy (TEM) studies of all the samples were performed as well. As it can be seen in Fig. 9a and b, randomly ordered Ter-POSS forms ill-defined compact aggregates while after complexation (Fig. 9c and d) some organizations typical of polymeric structures can be observed for all samples. Additional TEM images can be found in the $\mathrm{ESI} \dagger$ (Fig. S23, ESI $\dagger$ ).

\section{Experimental section}

\section{Materials and method}

The chemicals were purchased from the following sources: sodium hydroxide, PEG 300, 2-acetylpyridine, concentrated aqueous solution of ammonia, OPS (octa-phenyl silsesquioxane), iodine monochloride solution, sodium metabisulfite, anhydrous sodium sulphate, tetrakis (triphenylphosphine) palladium, copper(I) iodide, iron(II) triflate, zinc triflate, copper(II) triflate, and europium(III) triflate from Sigma-Aldrich; anhydrous tetrahydrofuran (THF) and 4-ethynylbenzaldehyde from TCI; and absolute EtOH and diisopropylamine from Fischer Chemical. Dichloromethane (DCM) and acetonitrile $\left(\mathrm{CH}_{3} \mathrm{CN}\right)$ used for the UV-Vis and fluorimeter spectroscopy measurements were of spectroscopic grade and were purchased from Carl Roth.

Quantitative ${ }^{1} \mathrm{H}$ NMR experiments were performed using a Varian VNMRS spectrometer operating at $9.4 \mathrm{~T}(400 \mathrm{MHz}$ for ${ }^{1} \mathrm{H}$ ) equipped with a $5 \mathrm{~mm}$ broadband probe, using the following acquisition parameters: a relaxation delay of $8.0 \mathrm{~s}$, an acquisition time of $2.0 \mathrm{~s}$, an excitation pulse of $90^{\circ}$, and 32 transients. Solid state ${ }^{13} \mathrm{C}$ and ${ }^{29} \mathrm{Si}$ NMR spectra were recorded 
at room temperature using a Bruker Avance-500 spectrometer operating at $11.7 \mathrm{~T}\left(125.7 \mathrm{MHz}\right.$ for ${ }^{13} \mathrm{C}$ and $99.3 \mathrm{MHz}$ for $\left.{ }^{29} \mathrm{Si}\right)$ using a $4.0 \mathrm{~mm}$ probe and spinning frequencies of 8 and $10 \mathrm{kHz}$. Combustion chemical analysis (C, H, N) was performed using a Thermo Finnigan Flash-45 EA1112 apparatus. UV-Vis measurements were performed using a Cary 5000 spectrophotometer (Varian) and fluorescence measurements using a Cary Eclipse instrument (Agilent technologies). The measurements were carried out using $10 \mathrm{~mm}$ Suprasil quartz cuvettes from Helma Analytics. Transmission electron microscopy images were recorded using a PHILIPS TECNAI 10 instrument at $80 \mathrm{keV}$.

\section{Synthesis I-POSS}

I-POSS was synthesized as previously reported. ${ }^{37}$ Briefly, $290 \mathrm{~mL}$ of $1.0 \mathrm{M} \mathrm{ICl} / \mathrm{CH}_{2} \mathrm{Cl}_{2}$ solution under $\mathrm{N}_{2}$ flow was cooled to $-40{ }^{\circ} \mathrm{C}$ and $26.7 \mathrm{~g}$ of OPS was added to the solution and the reaction was stirred for $24 \mathrm{~h}$ at $-40{ }^{\circ} \mathrm{C}$, warmed to room temperature for $24 \mathrm{~h}$, and then, quenched with $300 \mathrm{~mL}$ of sodium metabisulfite. Then, the organic layer was extracted, washed three times with water, filtered and dried over anhydrous sodium sulfate. The solution was dried under vacuum and a white solid was obtained. This solid was dissolved using THF and precipitated using cold methanol. ${ }^{29} \mathrm{Si}$ NMR: $\delta-77.9$. (Yield: $90 \%$, yield of para-IPOSS: $20 \%$ )

\section{Synthesis of $4^{\prime}$-(4-ethylnylphenyl)-[2,2':6,2 $\left.2^{\prime \prime}\right]$ terpyridine}

This terpyridine was synthesized as previously reported. ${ }^{19}$ In this case, $7.68 \mathrm{mmol}$ of $\mathrm{NaOH}$ was added to $10 \mathrm{~mL}$ of PEG 300 at $0{ }^{\circ} \mathrm{C}$. After this, $7.68 \mathrm{mmol}$ of 2-acetylpyridine was added. After $10 \mathrm{~min}$ of magnetic stirring, $3.38 \mathrm{mmol}$ of 4-ethynylbenzaldehyde was added and the solution was stirred at $0{ }^{\circ} \mathrm{C}$ for $4 \mathrm{~h}$. Then, $10 \mathrm{~mL}$ of concentrated aqueous NH3 solution was added and the suspension was stirred at room temperature overnight. Next day, the solid was isolated by filtration and washed with water and cold ethanol.

\section{Synthesis of novel Ter-POSS via a Sonogashira reaction ${ }^{32}$}

I-POSS (250 mg, $0.12 \mathrm{mmol}$ ), $4^{\prime}$-(4-ethylnylphenyl)-[2,2':6,2 $\left.2^{\prime \prime}\right]$ terpyridine (400 mg, $1.2 \mathrm{mmol}$ ), copper(I) iodide (16 mg, $0.08 \mathrm{mmol}$ ), and tetrakis(triphenylphosphine) palladium $(80 \mathrm{mg}$, $0.06 \mathrm{mmol}$ ) were added to a dry flask and purged with $\mathrm{N}_{2}$. Then, diisopropylamine $(10 \mathrm{~mL})$ and dry THF $(25 \mathrm{~mL})$ were added and the medium was purged again with $\mathrm{N}_{2}$. The reaction mixture was heated at boiling point for 7 days under $\mathrm{N}_{2}$ atmosphere. After 7 days, the reaction was filtered and the liquid was evaporated under vacuum. Then the solid was washed with water, several times with ethanol and with acetone. Finally, the solid was dried in a vacuum oven. ${ }^{29} \mathrm{Si}$ NMR: $\delta-80.69$. Elemental analysis (\%) of Ter-POSS: calcd: C, 76; H, 4; N, 9.2. Found: C, 75.6; $\mathrm{H}, 4.1 ; \mathrm{N}, 9.1$.

\section{Theoretical calculations}

Ter-POSS and O-POSS have been built from the reported X-ray structure of 1,3,5,7,9,11,13,15-octakis(4-(phenylethynyl)-phenyl)pentacyclo[9.5.1.13,9.15,15.17,13] octasiloxane. $^{41}$ A conformational search has then been performed on each molecule to get the most stable structures by coupling molecular mechanics (MM) and molecular dynamics (MD) simulations. All MM/MD calculations have been performed with the Materials Studio (MS) 2018 package using a force field derived from the Dreiding force field, in which (i) the van der Waals radius of the hydrogen atoms has been set to $2.83 \AA_{;}^{42}$ (ii) the electrostatic term follows Coulomb's law $(r-1)$ instead of the $r^{-2}$ default potential; (iii) an harmonic constraint $\left(d=4.82 \AA\right.$ and $k=100 \mathrm{kcal} \mathrm{mol}^{-1} \AA^{-2}$ ) has been defined on the distance between the nitrogen atoms of the two terminal pyridine rings of each arm to mimic the presence of a cation; (iv) the octasiloxane core has been geometrically-frozen during all simulations and (v) all the atomic charges have been set to COMPASS atomic charges.

The conformational search procedure to extract the most stable structure involves a geometry optimization at the MM level for each molecule followed by successive quenched-MD runs at increasing temperature during which the generated structures are systematically optimized and their energies compared. The procedure is stopped when the energy of the most stable conformer no longer decreases between successive quenched-MD runs. ${ }^{43}$ To characterize the dynamic character of the structures, 10 ns-MD simulations ( $N V T$ - snapshots saved every $10 \mathrm{fs}$ ) at room temperature have been performed from which radial distribution functions between the nitrogen atoms of the central pyridine ring of different terpyridine arms are built from the 1000 structures generated.

\section{Conclusions}

The synthesis of a novel POSS-based ligand bearing eight rigid and directional terpyridine arms (Ter-POSS) was successfully achieved via a Sonogashira coupling reaction. Ter-POSS was thoroughly characterized by employing a combination of different techniques including ${ }^{1} \mathrm{H},{ }^{13} \mathrm{C}$ and ${ }^{29} \mathrm{Si}$ NMR as well as FT-IR, UV-Vis and fluorescence spectroscopies. The selfassembly of the nano-caged molecular bricks in the presence of different transition metal ions $\left(\mathrm{Fe}^{2+}, \mathrm{Zn}^{2+}\right.$ and $\left.\mathrm{Cu}^{2+}\right)$ as well as the lanthanide $\left(\mathrm{Eu}^{3+}\right)$ was investigated via absorption and emission spectroscopies. Titration experiments revealed that four equivalents of metal cations are required to fully coordinate the Ter-POSS ligand. Additional insights into the structural organization of the POSS-based moieties in the presence of metal cations were obtained via molecular mechanics and molecular dynamics simulations. The results of this investigation perfectly correlate with the experimental findings. An in-depth TEM analysis allows supporting further the formation of 3D self-assembled polymeric organization. The final materials display an evident emission in different regions of the visible spectrum. This emission can be easily tuned as a function of the metal cation employed. Interestingly, the solid obtained through the coordination of $\mathrm{Eu}^{3+}$ displays a white light emission which can be ascribed to the presence of a combination of different contributions located in the blue, green and red regions of the fluorescence spectrum. The white-light emitting europium-based nanostructures exhibit 
one of the highest quantum yields reported in the literature for similar solids.

\section{Author contributions}

Andrea Santiago-Portillo: investigation (synthesis, characterization, and photophysical studies) and writing - original draft; Valerio Cinà: investigation (preparation of the I-POSS building unit); Esther Carbonell: supervision, discussion: the photophysical part, and validation; Luca Fusaro: NMR investigation; Vincent Lemaur: investigation (theoretical studies) and contribution in writing; Roberto Lazzaroni: investigation (Theoretical studies), contribution in writing, data validation, supervision and funding acquisition; Michelangelo Gruttadauria: validation and writing review \& editing; Francesco Giacalone: conceptualization, supervision, validation, writing review \& editing and funding acquisition; Carmela Aprile: conceptualization, supervision, validation, writing review \& editing and funding acquisition.

\section{Conflicts of interest}

There are no conflicts to declare.

\section{Acknowledgements}

Andrea Santiago Portillo acknowledges the University of Namur for postdoctoral fellowship. The authors acknowledge the FNRSFSR for the financial support. This research used resources of the Technological Platform "Physico-Chemical Characterization"-PC2 located at the University of Namur. The NamurMons collaboration is supported by the European Regional Development Fund (FEDER) and the Walloon Region (LCFM Low Carbon Footprint Materials - BIORG-EL project). Research in Namur is also supported by Fonds National de la Recherche Scientifique (F. R. S.-FNRS), grant number U.G014.19. Mons is also supported by the Fonds National de la Recherche Scientifique (F. R. S.-FNRS), under grant 2.5020.11 'Consortium des Équipements de Calcul Intensif (CÉCI)', and the Walloon Region, under grants 1117545 (Tier-1 supercomputer of the Fédération Wallonie-Bruxelles) and 1510616 (SOLIDYE project). V. C. gratefully acknowledges the University of Palermo and University of Namur for a co-funded PhD fellowship.

\section{Notes and references}

1 H. Shi, J. Yang, M. You, Z. Li and C. He, ACS Mater. Lett., 2020, 2, 296-316.

2 H.-B. Yao, M.-R. Gao and S.-H. Yu, Nanoscale, 2010, 2, 323-334.

3 I. Pramudya, C. G. Rico, C. Lee and H. Chung, Biomacromolecules, 2016, 17, 3853-3861.

4 R. Martínez-Máñez, F. Sancenón, M. Biyikal, M. Hecht and K. Rurack, J. Mater. Chem., 2011, 21, 12588-12604.

5 C. Calabrese, C. Aprile, M. Gruttadauria and F. Giacalone, Catal. Sci. Technol., 2020, 10, 7415-7447.
6 Y.-X. Zhao, B. Xu, X.-L. Ding and S.-G. He, J. Nanosci. Nanotechnol., 2013, 13, 819-823.

7 A. Arvinte, A. M. Sesay, V. Virtanen and C. Bala, Electroanalysis, 2008, 20, 2355-2362.

8 K. Tanaka, F. Ishiguro and Y. Chujo, J. Am. Chem. Soc., 2010, 132, 17649-17651.

9 H. Zhou, Q. Ye and J. Xu, Mater. Chem. Front., 2017, 1, 212-230.

10 X. Wang, D. Li, F. Yang, H. Shen, Z. Li and D. Wu, Polym. Chem., 2013, 4, 4596-4600.

11 H. Ghanbari, B. G. Cousins and A. M. A. Seifalian, Macromol. Rapid Commun., 2011, 32, 1032-1046.

12 L. Tang and Z. Qiu, J. Nanosci. Nanotechnol., 2016, 16, 10015-10020.

13 F. Zou, H. Ling, L. Zhou, F. Wang and Y. Li, Dyes Pigm., 2021, 184, 108840.

14 J. Duszczak, K. Mitula, A. Santiago-Portillo, L. Soumoy, M. Rzonsowska, R. Januszewski, L. Fusaro and C. Aprile, ACS Appl. Mater. Interfaces, 2021, 13, 22806-22818.

15 S. Köytepe, M. H. Demirel, A. Gültek and T. Seçkin, Polym. Int., 2014, 63, 778-787.

16 H. Hofmeier and U. S. Schubert, Chem. Soc. Rev., 2004, 33, 373-399.

17 H.-L. Au-Yeung, S. Y.-L. Leung, A. Y.-Y. Tam and V. W.-W. Yam, J. Am. Chem. Soc., 2014, 136, 17910-17913.

18 A. Wild, A. Winter, F. Schlutter and U. S. Schubert, Chem. Soc. Rev., 2011, 40, 1459-1511.

19 A. Winter, D. A. M. Egbe and U. S. Schubert, Org. Lett., 2007, 9, 2345-2348.

20 E. Carbonell, L. A. Bivona, L. Fusaro and C. Aprile, Inorg. Chem., 2017, 56, 6393-6403.

21 V. Cinà, E. Carbonell, L. Fusaro, H. García, M. Gruttadauria, F. Giacalone and C. Aprile, ChemPlusChem, 2020, 3, 391-398.

22 T. Zhang, J. Wang, M. Zhou, L. Ma, G. Yin, G. Chen and Q. Li, Tetrahedron, 2014, 70, 2478-2486.

23 S. Marchesi, C. Bisio, E. Boccaler and F. Carniato, ChemPlusChem, 2020, 85, 176-182.

24 J. Ohshita, M. Nakamura, K. Yamamoto, S. Watase and K. Matsukawa, Dalton Trans., 2015, 44, 8214-8220.

25 B. Dudziec, P. Zak and B. Marciniec, Polymers, 2019, 11, 504-543.

26 M. Nowacka, T. Makowski and A. Kowalewska, Materials, 2020, 13, 4491.

27 S. Pandey, A. Kumar Sharma, J.-L. Guo, N. Sharma and H.-F. Wu, ACS Sustainable Chem. Eng., 2020, 8, 9955-9961.

28 G. Li and J. Shinar, Appl. Phys. Lett., 2003, 83, 5359-5361.

29 S.-L. Lai, W.-Y. Tong, S. C. F. Kui, M.-Y. Chan, C.-C. kwok and C.-M. Che, Adv. Funct. Mater., 2013, 23, 5168-5176.

30 E. S.-H. Lam, D. P.-K. Tsang, W. H. Lam, A. Y.-Y. Tam, M.-Y. Chan, W.-T. Wong and V. W.-W. Yam, Chem. - Eur. J., 2013, 19, 6385-6397.

31 R. S. Deshpande, V. Bulovic and S. R. Forrest, Appl. Phys. Lett., 1999, 75, 888-890.

32 G. Zhao, Y. Zhu, S. Guang, F. Ke and H. Xu, New J. Chem., 2018, 42, 555-563.

33 X. Yang, J. D. Froehlich, H. S. Chae and S. Li, Adv. Funct. Mater., 2009, 19, 2623-2629. 
34 A. Winter, C. Friebe, M. Chiper, U. S. Schubert, M. Presselt, B. Dietzek, M. Schmitt and J. Popp, ChemPhysChem, 2009, 10, 787-798.

35 T. Suzuka, M. Adachi and K. Ogihara, Trans. Mater. Res. Soc. Jpn., 2015, 40, 103-106.

36 C.-C. Cheng, Y.-L. Chu, C.-W. Chu and D.-J. Lee, J. Mater. Chem. C, 2016, 4, 6461-6465.

37 M. F. Roll, M. Z. Asuncion, J. W. Kampf and R. M. Laine, ACS Nano, 2008, 2, 320-326.

38 G. Bechara, N. Leygue, C. Galaup, B. Mestre-Voegtlé and C. Picard, Tetrahedron, 2010, 66, 8594-8604.
39 R. Shunmugam and G. N. Tew, Polym. Adv. Technol., 2007, 18, 940-945.

40 Y. Q. Li, J. L. Bricks, U. Resch-Genger, M. Spieles and W. Rettig, J. Phys. Chem. A, 2006, 110, 10972-10984.

41 M. F. Roll, J. W. Kampf and R. M. Laine, Macromolecules, 2011, 44, 3425-3435.

42 S. Hoyas, V. Lemaur, Q. Duez, F. Saintmont, E. Halin, J. De Winter, P. Gerbaux and J. Cornil, Adv. Theory Simul., 2018, 1, 1800089-1800103.

43 V. Lemaur, J. Cornil, R. Lazzaroni, H. Sirringhaus, D. Beljonne and Y. Olivier, Chem. Mater., 2019, 31, 6889-6899. 